\title{
¿Es la universidad un entorno accesible? Historias de vida de estudiantes con discapacidad
}

\author{
Is the university an accessible environment? Life Stories of \\ Students with Disabilities
}

\author{
Almudena Cotán Fernández \\ Universidad Isabel I
}

Recibido: 08/03/2018

Aceptado: 28/06/2019

\begin{abstract}
This paper reflects the partial results of a doctoral thesis carried out under a research project financed by the Ministry of Economy and Competitiveness, which aims at analysing the main enablers/facilitators and obstacles that students with disabilities encountered during their stay in institutions of Higher Education. Based on a biographical-narrative method, the aim of this article is to identify and describe, according to the opinionof students with disabilities, the main barriers and aids perceived by a group of students with disabilities at the university, specifically those referring to architectural spaces. In order to collect the information, several instruments such as biographical interviews and photographs were used.Some of the main conclusions include the lack of accessibility in university environments owing to the presence of various elements that are not flexible when using them.
\end{abstract}

KEY WORDS: Disability, Higher Education, Biographical-narrative methodology, accessibility, Universal Design.

\section{RESUMEN}

Este artículo refleja los resultados parciales de una tesis doctoral vinculada a un proyecto de investigación financiado por el Ministerio de Economía y Competitividad, en el que se pretendía dar a conocer los principales facilitadores y obstaculizadores que los estudiantes con discapacidad identificaron durante su estancia en las instituciones de educación superior. Basado en un método biográfico-narrativo, en este artículo en concreto, se pretende identificar y describir, desde la vOz de los estudiantes con discapacidad, las principales barreras y ayudas que un grupo de estudiantes con discapacidad perciben en la universidad, concretamente las referidas a los espacios arquitectónicos. Para la recogida de información se ha hecho uso de diversos instrumentos como son las entrevistas biográficas o las fotografías. Entre las conclusiones obtenidas se destaca la falta de accesibilidad en los entornos universitarios existiendo elementos inadaptados y no siendo flexibles en su uso.

PALABRAS CLAVE: Discapacidad, Enseñanza Superior, metodología biográfica-narrativa, accesibilidad, Diseño Universal.

\section{Introducción}

Los estudiantes no tradicionales en las instituciones de Educación Superior (ES) han supuesto un auge importante en las últimas décadas (Seale, Georgeson, Mamas y Swain, 2015). Atendiendo a los datos ofrecidos por el Instituto Nacional de Estadística (INE) de las Enseñanzas Universitarias en España, en el curso 2017/2018 se matricularon un total de 1.575.579 de estudiantes en titulaciones universitarias. Este dato supone un incremento de 0,7\% frente al curso anterior, en el que había 1.564.943 estudiantes matriculados. De este colectivo de estudiantes, siguiendo los datos ofrecidos por la Fundación Universia (2018), se estima que el 1,5\% de ellos presenta algún tipo de discapacidad, siendo un total de 22.190 universitarios con discapacidad matriculados en 2018. Esta cifra supone un aumento del 6\% respecto al total de estudiantes matriculados en el curso 2016/2017 (20.773), y una cota máxima de alumnados con discapacidad matriculados en las instituciones de ES desde que se 
inició el registro de estudiantes con discapacidad en el 2008 por la Fundación Universia (Fundación Universia, 2018).

Estos datos han acentuado la elaboración de acciones, políticas y normas por parte de las instituciones de Educación Superior (ES) que permitan la participación plena de este colectivo de estudiantes en todos los espacios universitarios (Barnes 2007; Fuller, Bradley y Healey 2004;Hanafin, Shevlin, Kenny y Neela, 2007; Moriña, Cortes, y Melero 2014; Moswela y Mukhopadhyay 2011; Sandoval, Simón y Márquez, 2019). A este aspecto, se han producido importantes avances legislativos que aseguran la igualdad de acceso y permanencia de los estudiantes con discapacidad en estas instituciones. Buena muestra de ello se puede encontrar a nivel internacional, con la Declaración Universal de Derechos Humanos (1948). Posterior a esta Convención le han seguido numerosos acuerdos que han establecido y garantizado el acceso sin discriminación de las personas con discapacidad en las instituciones universitarias. Cabe mencionar, entre otras, la Convención Internacional de los Derechos de las Personas con Discapacidad de 2006 o la Estrategia Europa 2020 para un crecimiento inteligente, sostenible e integrador (2014), entre otros.

En el contexto nacional, el momento clave para los estudiantes con discapacidad llegó con el Real Decreto Legislativo 1/2013, el Real Decreto de Ordenación de las Enseñanzas Universitarias Oficiales (2007) o Ley Orgánica 4/2007, de 12 de abril (LOMLOU). Desde estas leyes, se reclama responder a las necesidades y demandas de este colectivo así como adoptar medidas que garanticen, entre otros aspectos, la adaptación de las pruebas de acceso, su permanencia en los estudios universitarios, exención de tasas, aumento de becas y ayudas al estudio, la creación espacios arquitectónicos accesibles, adaptación de los aspectos formativos y la integración plena de los estudiantes con discapacidad como comunidad universitaria (Aguirre y Miguel, 2011; Moriña y Cotán, 2017; Peralta, 2007; Rodríguez y Álvarez, 2014; Vander, 2015).

Así, el aumento y apuesta por la calidad en las instituciones de Educación Superior en materia atención a la diversidad es una realidad que va asentándose e incrementándose de forma notable en España. Sin embargo, ¿es esa la realidad que viven los alumnos con discapacidad en las instituciones universitarias? Aunque se puede afirmar que, tanto a nivel internacional como nacional la política y legislación educativa, ha avanzado de forma notoria hacia programas de mejora de la diversidad, autores como Oliver y Barnes (2010), Fernández, Álvarez y Malvar (2012) o Sandoval, Simón y Márquez (2019), evidencian cómo siguen existiendo centros de ES pocos inclusivos. Y, pese a ser instituciones cuya ratio en materia de alumnado con discapacidad se ha visto incrementada en los últimos años (Hadjikakou, Polycarpou y Hadjilia, 2010; Moriña y Morgado, 2018; Geogerson, Mamas y Swain, 2015), todavía sigue quedando un largo camino por recorrer (Moriña, 2017) para conseguir el desarrollo de prácticas inclusivas en la ES.

Barreras como la falta de formación del profesorado en materia de discapacidad, metodologías innacesibles, ausencia de sensibilización o planes de estudios inadaptados, son algunos de los obstáculos con los que este colectivo de estudiantes han de enfrentarse durante su trayectoria universitaria (Moriña y Morgado, 2018; Moswela y Mukhopadhyay 2011; Riddell, Tinklin y Wilson, 2002; Sandoval, Simón y Márquez, 2019; Shevlin, Kenny y Mcneela 2004). Obstáculos que, en ocasiones, son determinantes para que abandonen sus estudios universitarios (López-Gavira y Moriña, 2015). Y es que, cuando se habla de problemas de accesibilidad en los entornos universitarios, no sólo se alude a las instalaciones e infraestructuras (bibliotecas, aulas, despachos, comedor, zonas deportivas, espacios comunes, etc.), sino también al equipamiento que ofrecen las instalaciones (ordenador, dispositivos, mobiliario, iluminación, etc.) así como al personal docente, administrativos y servicios (actividades, seminarios, investigación, aprendizaje, etc.) que se ofrecen en ella (Fundación ONCE, 2010).

Sin embargo, y pese a ser una realidad estudiada y reflejada en diversos trabajos (Borland y James, 1999; Castellana y Sala, 2006; Fernández et al., 2012; Fuller et al. 2004; Luque, Rodríguez y Romero, 2005; Polo y López, 2005; Moriña y Morgado, 2018; Moswela y Mukhopadhyay 2011), mención aparte merece las barreras arquitectónicas. Frente al desarrollo de políticas y prácticas que conciben y diseñan espacios e infraestructuras accesibles para todo el alumnado como es el caso del Real Decreto Legislativo 1/2013 o la Convención sobre los Derechos de las Personas con 
Discapacidad, donde se garantiza la promoción de la accesibilidad universal, siguen existiendo numerosas barreras arquitectónicas que dificultan el acceso y participación de algunos estudiantes con discapacidad a determinados espacios (Sandoval, Simón y Márquez, 2019). Por ello, se considera imprescindible la supresión de barreras arquitectónicas en todos los espacios, productos o servicios. Esta se convertiría en una condición indispensable para que se cumpla el principio de igualdad de oportunidades y accesibilidad garantizándose, en todo momento, el respeto hacia la diversidad (Meyen 2015; Moriña y Morgado, 2018; Rodríguez, Suso, Vázquez y Velasco 2009). Tal y como indica Juncà (2003), permitiría que el alumnado viviera su experiencia universitaria con una completa autonomía personal aumentando los niveles de bienestar social e individual.

Bajo esta finalidad, las instituciones universitarias han de utilizar todos los recursos disponibles (Fernández et al., 2012). El uso y aplicación de los principios del Diseño Universal (Mace, 1997) en los diferentes espacios sería una cuestión indispensable a implementar para conseguir la accesibilidad física en las instituciones de ES. Tal y como nos especifican Silver, Bourke y Strehorn (1998) o Peralta (2007), la aplicación de estos principios no sólo beneficiarán al alumnado con discapacidad (por ejemplo, creación de rampas, señalización, fuentes bajas, puertas anchas, etc.), sino que beneficiaría a toda la población estudiantil (Pliner y Johnson, 2004).

Así, adoptar el Diseño Universal, tal y como se propuso en 1977 en el Centro de Diseño Universal en la Universidad de Carolina del Norte, permitiría a las instituciones de ES diseñar productos y entornos accesibles y utilizables sin que se requiera, posteriormente, de una adaptación o ajuste específico (Dell, Bell y Blackwell, 2015; Moriña y Morgado, 2018; Preiser y Smith, 2011; Vander, 2015; Watchorn, Larkin, Ang y Hitch, 2013). Esto facilitaría que estas instituciones pudieran ser comprensibles, utilizables practicable por un amplio grupo de personas bajo las características de seguridad, comodidad y autonomía. De esta forma, se crearían entornos educativos inclusivos donde sus características y condiciones fueran accesibles a un amplio rango de estudiantes. Tal y como afirma la Fundación ONCE (2010), las instituciones universitarias accesibles deben ser entornos en el que "los estudiantes no sólo puedan estar, sino en el que deseen estar" (p.40).

Bajo este marco conceptual y con el propósito de que las universidades se hagan eco de la situación de vulnerabilidad que experimentan este colectivo en sus instituciones, en es este artículo se pretende conocer, a través de la voz de dos estudiantes con discapacidad, el nivel de accesibilidad física de una institución de ES ubicadas en el sur de España. Para tal finalidad, de acuerdo con Moriña y Morgado (2018), Pliner y Johnson (2004) y Powell (2013), se parte de los principios del diseño universal en el que se analizara si la infraestructura de ambas instituciones presenta un uso equitativo, es flexible en su uso y los diseños son adaptados.

\section{Material y método}

Este artículo forma parte de una investigación más amplia (Cotán, 2015) y vinculada a un proyecto de investigación I+D+I financiado por el Ministerio de Educación y Ciencia de España (Barreras y ayudas que los estudiantes con discapacidad identifican en la Universidad, ref. EDU 201016264.). Este estudio, de cuatro años de duración (2010-2014) se realizó con un equipo multidisciplinar de la Universidad de Sevilla. El objetivo principal fue analizar las barreras y ayudas que los estudiantes con discapacidad identificaban en su trayectoria universitaria.

La metodología seleccionada para tal fin fue la biográfico-narrativa (Moriña, 2017), considerándola como una de las principales aportaciones del proyecto ya que permite dar voz y cabida a las experiencia de colectivos más vulnerables, como es el caso del alumnado con discapacidad (Hopkins, 2011). En esta investigación no sólo nos va a interesar la construcción de conocimientos nuevos, sino también poder desvelar y ofrecer el pensamiento y las experiencias de los estudiantes con discapacidad así como en las circunstancias que condicionan y dan forma al ambiente educativo universitario. Bajo esta metodología, y a través de las narraciones que surgen de las historias de vida, intentaremos conocer, investigar y analizar los diferentes contextos universitarios en los que los estudiantes están incluidos. En cualquier caso, el estudio de estos contextos universitarios ofrece una explicación de la cultura que envuelven a los estudiantes realizando una deconstrucción de la realidad 
a raíz de sus experiencias, reinterpretando los hechos sucedidos y transcurridos (Moriña y Cotán, 2017).

Así, nos acercaremos al tema de estudio analizando las experiencias y percepciones que los estudiantes con discapacidad tienen a ámbito institucional (accesibilidad en los diferentes entornos universitarios). De esta forma, el objetivo del presente trabajo se centra en identificar, describir y explicar las barreras y ayudas que dos alumnos con discapacidad visual y motora perciben en la universidad como institución.

Para tal finalidad, analizaremos los diferentes espacios arquitectónicos siendo este el punto fuerte del mismo al evidenciar las barreras existentes para que, desde las instituciones universitarias, se tomen las medidas que se consideren oportunas así como se generen procesos de sensibilización hacia este colectivo. Desde este punto de vista, pretendemos estudiar si la universidad ofrece entornos accesibles y usables por un amplio colectivo de estudiantes (en concreto el alumnado con discapacidad), si están adaptadas a la legislación actual así como a las necesidades del alumnado o, por el contrario, no hace uso de los principios del Diseño Universal y presenta barreras de accesibilidad arquitectónica y física en sus diferentes espacios. Es decir, queremos conocer los principales facilitadores y obstaculizadores que este colectivo de participantes identifican en la institución universitaria a nivel arquitectónico durante su estancia en la misma.

\subsection{Recogida de datos}

Para dar respuesta al objetivo de investigación que presentamos en este artículo, la recogida de información se ha realizado en torno a dos áreas de conocimientos: Ciencias e Ingeniería y Ciencias Sociales y Jurídicas.

Los hallazgos reflejados en este trabajo han sido recogido durante cinco años (2010-2015) a través de las tres fases del proyecto de investigación. En un primer momento, se organizaron grupos focales por áreas de conocimientos así como entrevistas individuales que se realizaron de forma oral y escrita. En esta fase inicial, participaron un total de 44 estudiantes. Los instrumentos de investigación utilizados fueron las entrevistas individuales y grupales así como grupos de discusión. En un segundo momento y fase, participaron 16 estudiantes para realizar microhistorias de vida caracterizadas por ser temáticas (Sandín, 2003). Los instrumentos de datos empleados en esta fase fueron tres: líneas de vida, entrevistas semiestructuradas y autoinforme. Para finalizar, en la última y tercera fase del proyecto se pasó a profundizar en las historias de vida en profundidad de ocho estudiantes. Para la elaboración de las historias de vida se utilizaron la polifonía de voces (Frank, 2011). Las técnicas empleadas en esta fase fueron variadas y diversas: entrevistas semiestructuradas, entrevistas biográficas, entrevistas con personas claves en sus experiencias universitarias, observaciones, notas de campo, fotografías y la técnica de observación "Un día en la vida de...".

Los datos que reflejamos en este artículos han sido extraídos de la tercera fase de investigación y se centran en analizar de forma exclusiva la arquitectura e infraestructura de las instituciones universitarias.

\subsection{Participantes}

Para la selección de los participantes de esta investigación, debemos remontarnos al curso académico 2009/2010, momento en el que tuvo lugar la primera toma de contacto con los participantes y coincidiendo con el inicio de la primera fase del proyecto. En el curso 2009/2010, podemos decir que el número de alumnado matriculado en la Universidad de Sevilla era de 72.358. De este colectivo de alumnado, 445 estudiantes con discapacidad estaban matriculados en este curso académico, lo que supone un $0,6 \%$ de la población estudiantil.

Para acceder a los participantes, y teniendo en cuenta la Ley de Protección de Datos "II del REAL DECRETO 1720/2007, de 21 de diciembre, por el que se aprueba el Reglamento de desarrollo de la Ley Orgánica 15/1999, de 13 de diciembre, de protección de datos de carácter personal", el primer paso a dar fue contactar (a través de una entrevista telefónica) con la responsable de la Servicio de Atención al Alumnado con Discapacidad (SA.D) y hacerle conocedora de nuestro proyecto de investigación y el objeto del contacto. 
Una vez realizado este paso, fue la responsable de la Unidad de Atención al Estudiante con discapacidad quien intermedió para solicitar la participación de los estudiantes, enviándole un email en el que se adjuntaba la carta de presentación donde se explicaba toda la investigación y se les invitaba a participar. Aquellos estudiantes que estuvieran interesados en participar sólo debían responder al email de contacto que se indicaba en el correo enviado por el responsable.

Para la selección de la muestra de participantes, se optó por una "selección basada en criterios" (Goetz y LeCompte, 1988), donde la selección estaba determinada previamente por unos criterios establecidos por los investigadores. Siguiendo a autores como Patton (1987) o Pujadas (2002), el muestreo selectivo se ha realizado en base a los siguientes rasgos, criterios y características relevantes: estudiante con discapacidad, accesibilidad, participación, disponibilidad para participar, relevancia, voluntariedad e interés o necesidad.

En este artículo de investigación se reflejan dos historias de vida de los tres estudiantes con discapacidad que participaron en la ya mencionada tesis doctoral (Cotán, 2015). De los dos participantes, Andrés y Rafael, sus edades estaban comprendidas entre los 27 y 20 años. Las titulaciones de pertenencia eran Ingeniería y Periodismo, con una permanencia media en la universidad de seis años. Para finalizar, los participantes presentaban una discapacidad visual (ceguera) y física (enfermedad de Duchenne).

\subsection{Análisis de los datos}

El análisis y tratamiento de los datos se realizó desde una doble perspectiva. En primer lugar, un análisis comparativo y estructural (Riessman, 2008) de toda la información recopilada. Para tal finalidad, se realizó un sistema de categorías y códigos propuestos por Miles y Huberman (1994). Por otro lado, se realizó un análisis narrativo (Goodley, Lawthom, Clough y Moore, 2004) construyendo las historias de vida de cada informante abarcando "temas sobre lo que se habla y el cómo se habla, quién, cuándo y por qué" (Cotán, 2016, p.202). Para la elaboración de las historias de vida se ha optado por un diseño multivocal polifónico (Mallimaci y Giménez, 2006) donde las voces de los participantes se cruzaron con los informantes claves previamente seleccionados por ellos mismos.

Siguiendo en todo momento un punto de vista interpretativo, al escribir las historias de vida se plantearon tres aspectos principales (Leíte, 2011): 1. Fidelidad del relato del protagonista; 2. Marco de re-construcción de la narración y, 3. Línea cronológica del relato. Se optó por una postura reflexiva y comprensiva sobre la experiencia de los estudiantes enmarcándola dentro del contexto social, cultural y político en el que se desarrollaba. Así, las historias de vida se realizaron a través de un análisis dialógico y crítico donde el protagonista analizaba todos los sucesos acontecidos en su trayectoria.

En este artículo presentamos los datos centrados en barreras arquitectónicas y obtenidos a través del análisis narrativo de Rafa y Andrés. Para ello, apoyaremos el discurso de nuestros protagonistas con las narraciones de otros informantes claves y con fotografías tomadas por ellos mismos con una breve descripción.

\subsection{Consentimiento informado}

Desde el inicio de la investigación, los participantes tuvieron en su poder el consentimiento informado donde se les informaba del objetivo de investigación, así como del uso y tratamiento de los datos. Durante el transcurso del proyecto, los participantes tenían acceso a la información recogida pudiendo añadir, suprimir o modificar la información que ellos considerasen oportuna. Asimismo, aquellos estudiantes que decidieron declinar su participación durante la recogida de información, se les informó que sus datos no serían incorporados, tratados ni publicados con posterioridad. 


\section{Resultados}

Los resultados que a continuación se presentan versan sobre las barreras arquitectónicas y de infraestructuras que dos estudiantes con discapacidad identificaron en sus universidades de procedencia. Para tal fin, nos hemos centrado en la propuesta de análisis de Moriña y Morgado (2018, p.16) tomando como referencia a Luque et al. (2005).

Así, las barreras que en este trabajo se van a representar quedan aglutinadas en tres dimensiones:

1. Construcción de barreras: Dentro de los edificios y espacios universitarios (pasillos estrechos, escaleras, rampas, baños adaptados, etc.).

2. Barreras ambientales: Muebles, condiciones ambientales, elementos del aula (escritorios, sillas, luz, temperatura, ruido, etc.).

3. Barreras de comunicación: Señalización, acceso a la información (megafonía, señalización de escaleras o puertas, letreros, ordenadores no adaptados, PowerPoint, entornos visuales, etc.).

3.1. Cuando el entorno nos impone las barreras. Historia de vida de Rafa

La Universidad te da cosas buenas y cosas malas. Te hace enfrentarte a cosas que nunca imaginaste y eso enriquece, aunque en ocasiones nos desgaste y nos canse. Uno de los mayores obstáculos con los que me he encontrado en la universidad son las barreras arquitectónicas. Quizás, otro alumno con una discapacidad auditiva o psíquica no lo note tanto, pero yo, con una discapacidad motórica se me hace muy difícil acceder a ciertos espacios.

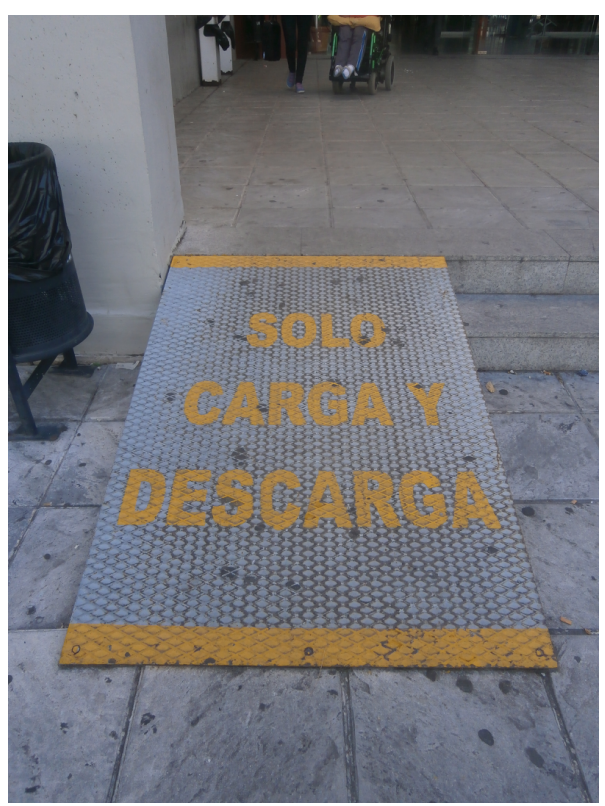

Imagen 1. Falta de acceso a la entrada principal de la facultad

"Aquí se puede ver la entrada principal de la Facultad. Es una rampa de carga y descarga. La he elegido para expresar la falta de acceso adecuado en la entrada para las personas que utilizamos silla de ruedas" (Rafa, técnica de la fotografía).

Las rampas en mi facultad no se realizaron ni se pensaron para personas en sillas de ruedas. Son rampas de carga y descarga, por lo que necesito de una persona que me ayude a subir y a bajar. Así que, al estar situada en la planta principal pues casi nadie se da cuenta, solo yo que soy el que la utilizo. Por lo que la evito y acabo accediendo a la facultad por el garaje o por el ascensor. 
Y bueno, el tema del ascensor y del baño también merecen que le haga una mención específica. $\mathrm{El}$ ascensor es el mismo para todos, no hay uno destinado para personas con discapacidad. En el cambio horario están completamente llenos y tengo que esperar casi un cuarto de hora para poder cogerlo y, claro está, ese tiempo de espera hace que llegue tarde a clase. Sin embargo, sí que puedo decir que las dimensiones del mismo respetan lo exigido por la normativa y pese a que tenga que entrar de cara y salir siempre de espaldas, puedo acceder sin ningún tipo de problema.

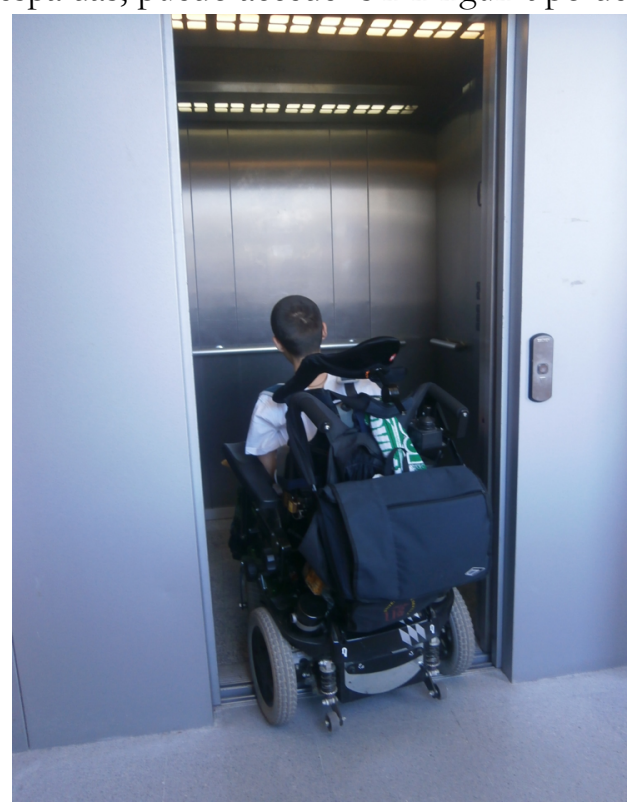

Imagen 2. Ascensores accesibles para una silla de ruedas

"En esta foto se observa que puedo acceder al ascensor sin ningún tipo de problema. Es totalmente imprescindible y necesario que los ascensores tengan unas dimensiones adecuadas para que pueda acceder con la silla de ruedas sin ningún tipo de dificultad" (Rafa, técnica de la fotografía).

Respecto al baño principal, lo primero que percibo al entrar son las puertas estrechas que tiene. No puedo girar ni acceder yo solo, por lo que mi madre tiene que realizar un gran esfuerzo al girar la silla para que pueda acceder al baño ya que la puerta es muy justa y rozo con mi silla de ruedas.

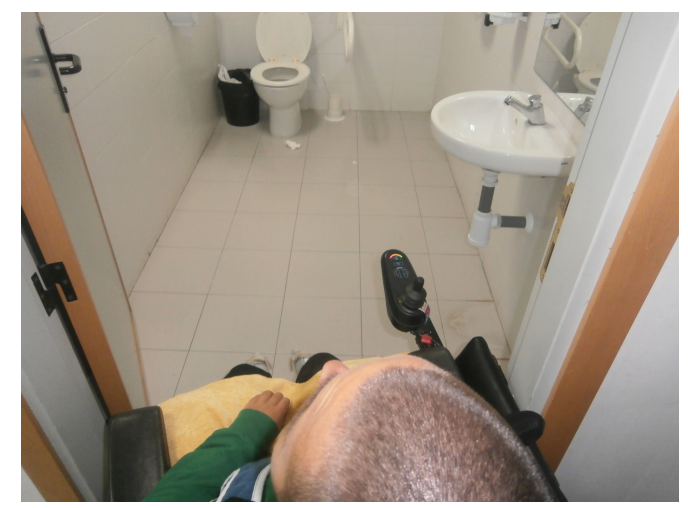

Imagen 3. Espacios estrechos y reducidos que dificultan la vida diaria a personas en silla de ruedas dentro del entorno de la Universidad 
"En estas fotografías se puede apreciar la estrechez de la puerta. La silla de rueda entra por milímetros. Esto me dificulta bastante el acceso al baño adaptado" (Rafa, técnica de la fotografía).

Después, en toda la facultad hay un servicio que se encuentra reservado para minusválidos pero ¿realmente es así? Yo he tenido que esperar fuera a que salieran compañeros que no lo necesitaban o tener que limpiar la taza del wc porque estaba sucia del mal uso. Quizás la solución podría ser poner una cerradura y darnos una llave a las personas que realmente lo necesitamos. Pero nos han dicho que no, que eso no puede ser. Mi madre, inclusive, ha llegado a pensar en poner un cartel para concienciar a los compañeros sobre mi situación, pero no sé si será la solución. Es lo mismo de siempre, la falta de concienciación.

Pero creo que el problema de la estrechez de las puertas no es sólo del baño, sino de toda la facultad. Además, aparte de que son estrechas, las puertas se abren y se cierran solas, no tienen ningún mecanismo para mantenerse abierta y eso, para las personas con mis necesidades es esencial ya que necesitamos tiempo para poder pasar.

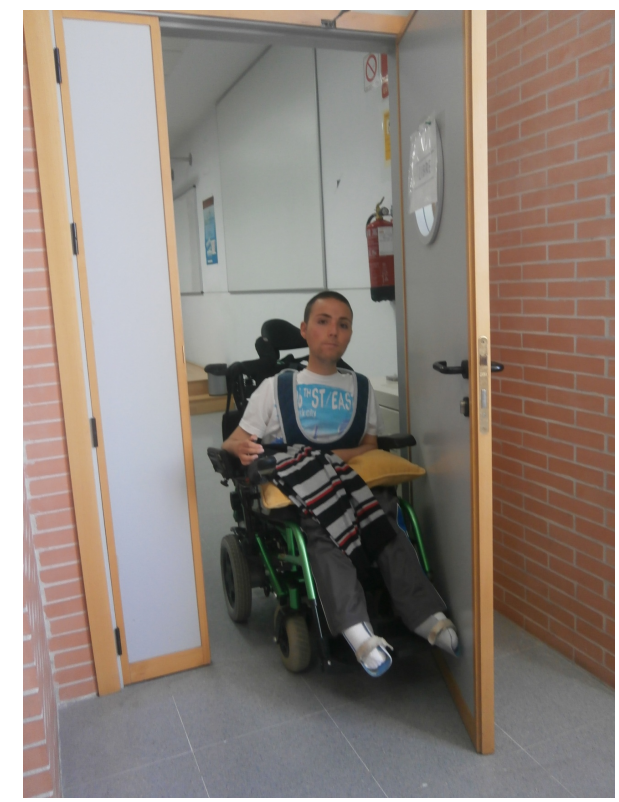

Imagen 4. Puertas sin sujeción

"En la sala de ordenadores, las puertas tienen un mecanismo para qué no se mantengan abiertas. Esto me dificulta salir con la silla de ruedas del aula así como la autonomía para poder salir o entrar, por lo que me veo obligado y en la necesidad de tener que pedir ayuda" (Rafa, técnica de la fotografía).

Los despachos de los profesores son tan pequeños que, en algunas ocasiones, me han tenido que atender fuera con la puerta abierta porque no he podido entrar con la silla de ruedas. Y, en las clases, no es que la situación sea diferente. Sí que es cierto que han adaptado determinadas cosas como, por ejemplo, el escritorio. Pero no lo han hecho desde mi punto de vista sino desde el suyo, es decir, lo que han hecho es cortar un trozo de banca para poder meter mi silla de ruedas pero no puedo moverme con libertad al no ser una mesa portátil.

"Las aulas donde está Rafael están adaptadas para que tenga su silla de ruedas, se ha cortado un trozo de la banca para que esté él... o sea, que se sabe que él está ahí y se 
hace algunas adaptaciones para él y otros alumnos que puedan estar en su situación." (María, profesora de Rafa. Entrevista semiestructurada).

Además, cuando me pongo en mi sitio (siempre es el mismo), no puedo girarme para ver al profesor cuando está explicando y si tengo el ordenador, tengo que mirar hacia el frente al no poder girar la cabeza, por lo que he optado en asistir a clase sin el ordenador y escuchar directamente las explicaciones del profesor, sin tomar ningún tipo de apuntes o esquemas como hacía cada vez que llevaba mi ordenador.

Y bueno, a la hora de realizar exposiciones, todos los compañeros se suben a la tarima del aula para exponer sus trabajos. La única forma de acceder a ésta es por escaleras y yo, como no me suban a pulso, no puedo, por lo que tengo que exponer desde abajo. Pienso que poner una rampa incrustada tampoco supondría tanto y, pese a que mi grupo se quede conmigo, eso no me hace sentir bien ni igual que los demás. Esa situación me hace sentirme raro, pero sobretodo, me hace sentir diferente a mis compañeros ya que yo no puedo exponer el trabajo igual que ellos. No es justo que por una barrera arquitectónica que tiene solución, tenga que sentirme diferente.

"Para exponer los trabajos, él no puede realizarlo igual que yo. Se tiene que quedar abajo y eso influye psicológicamente $\mathrm{y}$, a lo mejor, nosotros nos podemos abajo o a él lo ponemos en medio..." (Loreto, compañera de Rafa. Entrevista semiestructurada).

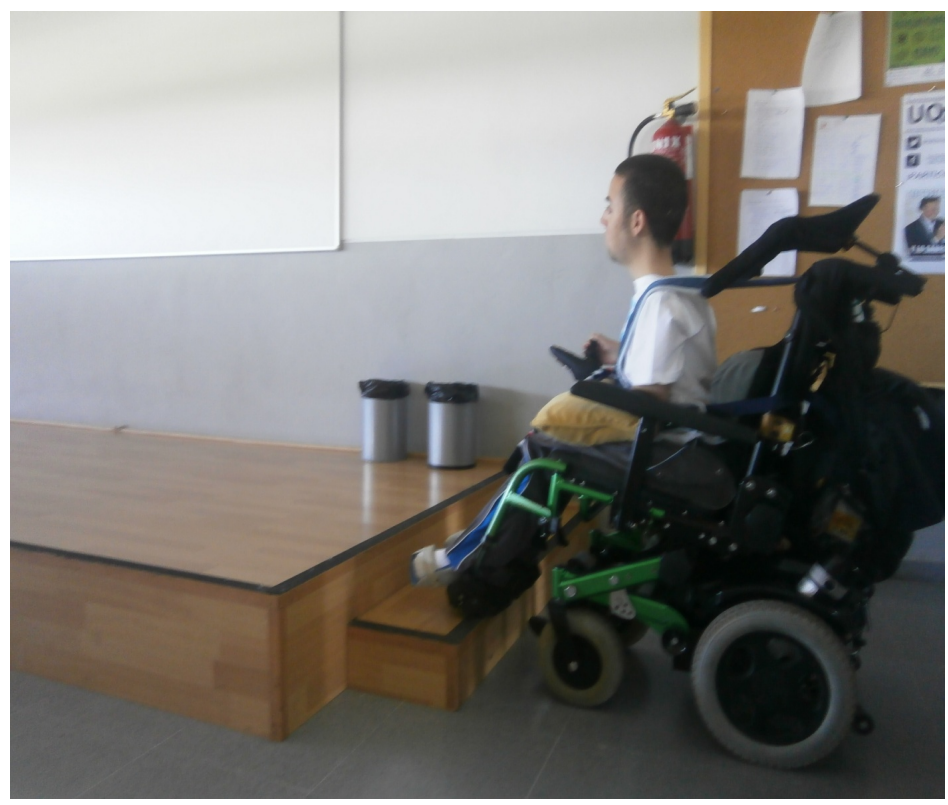

Imagen 5. Estrados no accesibles para personas en silla de ruedas

"Todas las Aulas de mi Universidad carecen de adaptaciones de los estrados para que persona como yo podamos subir a ellos. Esto provoca que no pueda realizar las exposiciones de los trabajos en igualdad de condiciones que el resto de alumnos" (Rafa, técnica de la fotografía).

En la sala de ordenadores la situación no es que sea muy diferente. Necesito de un ordenador adaptado con programa de voz y con un ratón táctil, pero no los hay, por lo que mis compañeros pueden escribir, hacer trabajos y seguir al profesor y yo, como no sea con mi ordenador personal que me lo tengo que costear, no puedo hacer lo mismo que ellos y, por lo tanto, hacer trabajo extra en mi casa. A su favor tengo que decir que hay espacios para poder ponerme con mi silla y mi ordenador. 
Aunque éste no sea el más adecuado ya que mi silla no entra y tengo que mantenerme a cierta distancia de la mesa.

"Si el lugar estuviera adaptado, Rafa no tiene ningún problema, él se busca la vida para que no le falte de nada, o sea, si no tuviera a su madre ya se encargaría de que estuviera su primo, yo o quien sea para que le ayudemos. Entonces, eso no es una limitación, la limitación está en lo físico, en los lugares" (Consuelo, madre de Rafa)

Pero volvemos a lo mismo de siempre, es la facultad, la sociedad o el contexto el que me impone las barreras y no me deja ser autónomo. Quizás si, a la hora de realizar las adaptaciones nos preguntaran, muchos de los problemas que tengo no existirían.

\subsection{Adaptaciones dependientes de la buena voluntad. Historia de vida de Andrés}

Han pasado ya seis años desde que empezara los estudios de ingeniería, y pese a que he tenido experiencias amargas, también cabe destacar que hay otras cosas que han merecido la pena. Son ejemplos de ello, el trabajo de una calidad superior del personal de conserjería y mantenimiento de la ETSII.

Este servicio se ha involucrado de una manera especial en la adaptación y el mantenimiento de las herramientas físicas que hacen que este tipo de barreras, no merezcan la pena considerarse. Son personas que están muy comprometidos con su trabajo, que te ayudan y que en cuanto ven que las cosas funcionan están al día siguiente pensando en otra cosa que pueda a ellos ayudarle incluso a mejorar el servicio.

"Entiendo que ayudar a los usuarios es algo que está implícito en nuestro trabajo. Personalmente creo que el personal de nuestra unidad, por distintos motivos, tiene una motivación que va más allá de la implicación profesional. Creo que tienen una actitud proactiva ante este tema." (Personal PAS).

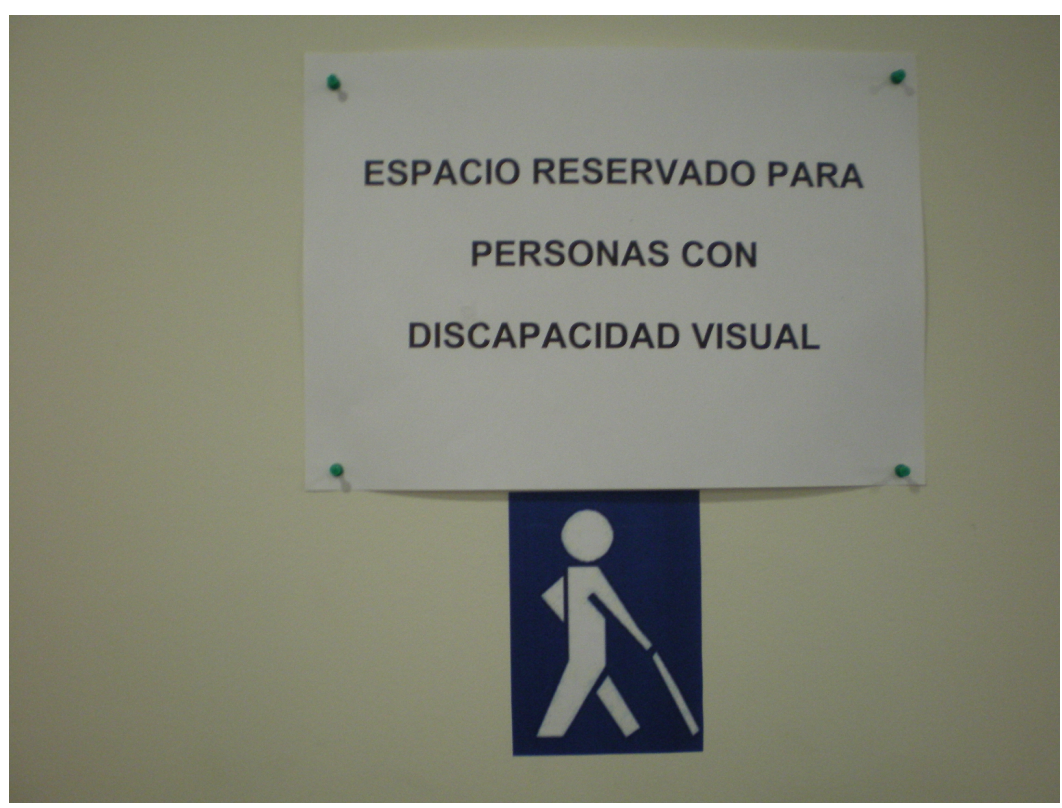

Imagen 6. Calidad en el espacio gracias a Conserjería

"Gracias a conserjería puedo sentarme tanto en primera fila de las clases como en otros espacios reservados de biblioteca" (Andrés, técnica de la fotografía). 
Entre otras actuaciones, destacan la señalización de escaleras, puertas, salvado de desniveles en mesas para un mejor manejo de los libros y cuadernos, reserva de puestos, electrificado de mesas, ampliación de red eléctrica con enchufes de cobertura en laboratorio, sustitución de carteles de ubicación con fuente mas ampliada, etc.

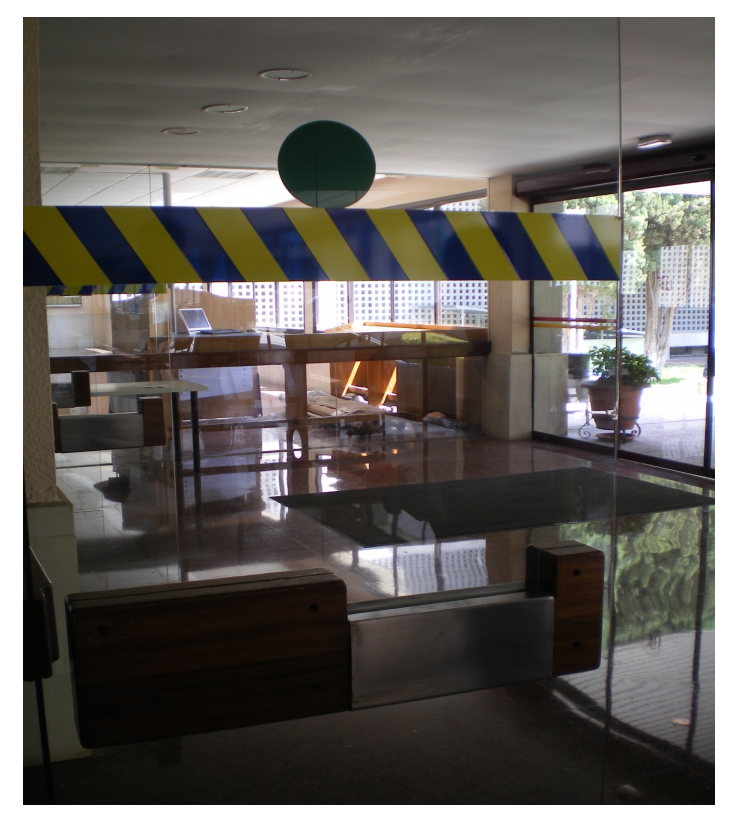

Imagen 7. Sin barreras

"Puntos de atención coloreados con tonos complementarios en puertas de cristal para poder acceder y salir del edificio sin problemas" (Andrés, técnica de la fotografía).

"Las adaptaciones han consistido básicamente en la eliminación de algún obstáculo, modificación de algún mobiliario, ubicación de algún dispositivo, iluminación de algún espacio o utilización de algún tipo de señalización" (Personal PAS. Entrevista estructurada).

El servicio de biblioteca me ha ofrecido toda clase de facilidades a la hora de acceder a contenidos, búsqueda de libros en papel, recomendación de materiales electrónicos en sustitución o complementarios a los anteriores, recomendaciones de títulos según su fuente y legibilidad... Además, la biblioteca de la Universidad de "suprimido para revisión anónima" ha adquirido una serie de lupas TV que se encuentran en las bibliotecas, para facilitar el acceso al material impreso, siendo la de informática una de las primeras en contar con este equipamiento. 


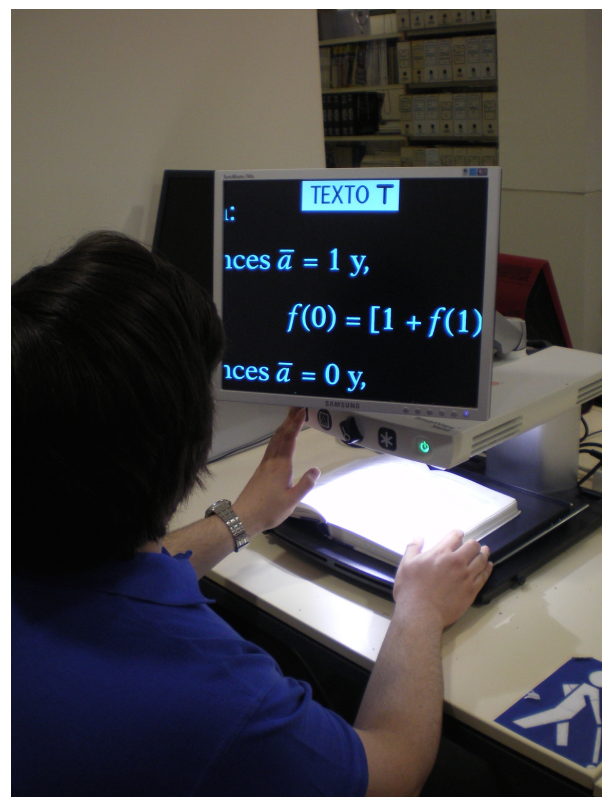

Imagen 8. Lupa TV

"La lupa TV se encuentra en la biblioteca en un espacio reservado y adaptado. Gracias a ella puedo leer y ampliar la información de los libros sin tener que ampliar los documentos ni invertir recursos por mi cuenta" (Andrés, técnica de la fotografía).

Incondicional la labor del perito de arquitectura de la Universidad, que ha llevado a cabo todas las acciones de adaptación del centro de estudios como de otros espacios de la universidad en un tiempo record y con unos resultados óptimos y excelentes. He conseguido que se hayan adaptado casi todos los edificios: sensores de apertura en los ascensores, pegatinas en las puertas de cristal, iluminación, aumento del tamaño de la letra en los letreros de los despachos, etc.

La verdad es que todo fue muy rápido y eficaz, en cuestión de mes y medio aproximadamente en colaboración con el servicio de conserjería estuvo todo solucionado. Se pusieron pegatinas en las puertas o pintaron los escalones de las escaleras. Eso fue una gran ayuda.

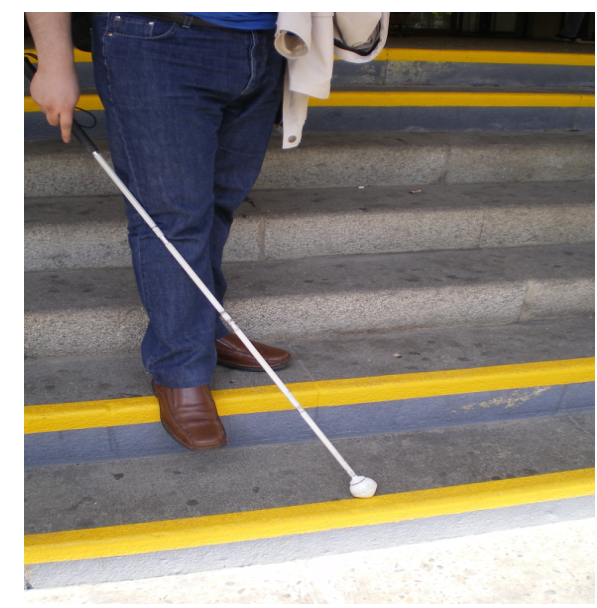

Imagen 9. Escalones accesibles 
"En esta fotografía se puede observar las líneas donde comienzan y finalizan la escalera que sirven de gran ayuda para poder acceder a la escuela" (Andrés, técnica de la fotografía).

Así también impecable el trabajo de tantos profesores, que "en la clandestinidad" han desarrollado metodologías para que pudiera acceder a las clases: me han facilitado los materiales de estudio, a los exámenes, a las prácticas, incorporando tutorías específicas, realizando exámenes asistidos para garantizar el acceso a los contenidos gráficos, esquemas, circuitos, etc.

"Cierto grupo de profesores también le ha ayudado mucho pues le ofrecían las transcripciones de códigos que se escribían en la pizarra al final de clase, las transparencias, tutorías personalizadas, también le ayudaban en los exámenes, etc." (Carlos, hermano Andrés. Entrevista semiestructurada).

También he de mencionar las ayudas recibidas a través de la ONCE como las lentes ópticas ${ }^{29}$ o la lupa digital ${ }^{30}$ que me permiten acceder tanto a la lectura de textos de pequeño tamaño como la lectura y acceso de la información de lo que se expone en la pizarra.

"La última ayuda que conozco es el proyector que le facilitó la ONCE para el tema de la pizarra porque no la veía bien. Entonces, él lo llevaba él a clase, se enganchaba con el ordenador y la veía estupendamente." (Mamen, compañera Andrés. Entrevista semiestructurada).

Como conclusión, solo puedo decir, que alcanzar objetivos solo se consigue cuando todas las partes están implicadas al mismo nivel en el mismo fin y sin excluir a nadie, pese a las ideas políticas o sociales que se puedan tener preconcebidas que solo generan prejuicios a la parte minoritaria. El futuro es ya hoy, del mañana nada se sabe y merece la pena mirar solamente hacia delante sin importar lo que pasa ahora. Nadie va a luchar por ti si tu no luchas primero.

\section{Discusión y conclusiones}

Numerosas universidades manifiestan el derecho de los estudiantes con discapacidad a participar en la vida universitaria en igualdad de condiciones que sus compañeros. No obstante, los resultados hallados en este trabajo reflejan que esta inclusión e igualdad de forma plena aún no se ha conseguido ya que en sus relatos se reflejan determinadas barreras con las que estos estudiantes se han ido encontrado en sus trayectorias. Buena muestra de ello, lo podemos localizar en las barreras arquitectónicas e infraestructura que los estudiantes indicaron. Estos resultados se encuentran también en otros estudios previos, como los de Borland y James (1999), Castellana y Sala (2006), Fernández et al., 2012; Hadjikakou et al., (2010), Hanafin et al. (2007), Luque et al., (2005), Moriña y Morgado (2008), Moswela y Mukhopadhyay (2011), Rodríguez et al. (2009) y Shevlin et al. (2004), donde encontraron barreras físicas y espacios inaccesibles en los centros universitarios.

Los participantes destacaron las barreras arquitectónicas como obstáculos que le dificultaban e impedían desarrollar sus experiencias con normalidad e igualdad. Entre las principales barreras incluidas dentro del campus universitario destacaron los escalones, puertas de cristal, ausencia de rampas, ascensores, letreros de los despachos, falta de rampas, mobiliario inadaptado, falta de

\footnotetext{
${ }^{29}$ Gafas de visión que permiten leer textos de tamaño pequeño.

${ }^{30}$ Lupa digital que se conecta al monitor del ordenador y permite ampliar el texto de la pizarra u otro recurso como libre, folio, etc.
} 
recursos, etc. Esto ratifica las palabras de Oliver y Barnes (2010) o Fernández et al. (2012) cuando afirman que siguen existiendo centros de ES pocos inclusivos.

Todas estas barreras dificultaron notablemente la vida del estudiante durante su estancia universitaria posicionándolos, además, en una doble situación de desventaja y discriminación frente a sus compañeros (Cotán, 2016). Además, en algunas ocasiones estas barreras le generaron una situación de dependencia. En especial, Rafa hizo hincapié en los problemas que tenía cuando quería acceder a los diferentes espacios de la universidad o cuando tenían que hacer uso de los aseos. Así, sería esencial la construcción del espacio desde los fundamentos y presupuestos del diseño universal construyendo entornos y contextos accesibles para todos, donde el alumnado pueda participar sin necesidad de adaptar ni realizar ningún proyecto ni diseño específico (Dell et al., 2015; Moriña y Morgado, 2018; Preiser y Smith, 2011; Vander, 2015; Watchorn et al., 2013).

Desde el punto de vista de los estudiantes con discapacidad visual cobra especial relevancia la correcta señalización que existe en algunos espacios ya que indicaron que esto es importante para acceder y leer información. En este sentido, investigaciones como las de Borland y James (1999), Castellana y Sala (2005), Fuller et al. (2004) y Polo y López (2005) coinciden con resultados similares en los que detallan que existen diversas barreras y que, en función del tipo de discapacidad, algunas se hacen más destacadas que otras. Estos obstáculos ratifican los presupuestos del modelo social de la discapacidad donde es el contexto el que genera las barreras a las personas con discapacidad huyendo así de la idea del modelo médico de discapacidad. En este sentido, tal y como apuntan Hopkins (2011) y Moriña y Morgado (2018), las dificultades con los que los estudiantes con discapacidad se encuentran en la universidad están en su entorno.

Esta realidad lleva a confirmar la necesidad de adaptación y reajuste en los centros de enseñanza superior para que sean totalmente accesibles para toda la comunidad universitaria (Cotán, 2016). Por ello, el objetivo que deben de seguir las políticas y los planes de mejora ha de ser alcanzar la inclusión plena, tomando como referencia el diseño universal. En este sentido, estas mejoras no sólo beneficiarán a los estudiantes con discapacidad sino al colectivo de estudiantes en general (Silver et al., 1998; Peralta, 2007; Pliner y Johnson, 2004).

En definitiva, los estudiantes indicaron que una universidad ideal debería ser más accesible, eliminado las barreras arquitectónicas y de comunicación. Destacaron además que, en muchos de los casos, se trata de cuestiones que pueden solucionarse fácilmente y sin necesidad de emplear muchos recursos. Sugirieron que lo ideal sería diseñar una universidad para todos, sin necesidad de adaptaciones específicas (Moriña y Morgado, 2018), donde todos se sintieran incluidos y evitar datos como los reflejados en esta investigación y en la de Luque et al., (2005), en los que se concluye que en los centros universitarios existen elementos inaccesibles (Cotán, 2016; Oliver y Barnes, 2010; Fernández et al, 2012). La meta debe ser, por tanto, alcanzar la plena inclusión de todos y todas, tomando como referente el diseño universal (Mace, 1997). Así, el alumnado con discapacidad podría vivir su experiencia con plena autonomía y participación en todos los espacios universitarios (Juncà, 2003).

El reconocimiento de todas estas propuestas y el fuerte compromiso por implementarlas, permitirá que estas sugerencias sean concretadas través de políticas, estrategias, procesos y programas, cuyo único fin sea la consecución de un verdadero espacio universitario inclusivo y de excelencia caracterizado por la igualdad de oportunidades y condiciones de acceso y permanencia de todo sus estudiantes.

\section{Referencias bibliográficas}

Aguirre, R. y Miguel, C. (2011). Estudiantes inmigrantes con diversidad funcional en la Universidad Complutense de Madrid: nuevos retos para la intervención del Trabajo Social. Revista de Trabajo y Acción Social, 50, 198-214. 
Barnes, C. (2007). Citizenship and vulnerability: Disability and issues of social and political engagement. Brital Journal Sociology, 58, 4, 717-718.

Boletín Oficial del Estado (2007). Ley Orgánica 4/2007, de 12 de abril, por la que se modifica la Ley Orgánica de 6/2001, de 21 de diciembre, de Universidades. Disponible en: https://bit.ly/2AgbqYt.

Boletín Oficial del Estado (2012). II del REAL DECRETO 1720/2007, de 21 de diciembre, por el que se aprueba el Reglamento de desarrollo de la Ley Orgánica 15/1999, de 13 de diciembre, de protección de datos de carácter personal. Disponible en: https://bit.ly/2JU10ou (2 de noviembre de 2018)

Boletín Oficial del Estado (2013). Real Decreto Legislativo 1/2013, de 29 de noviembre, por el que se aprueba el Texto Refundido de la Ley General de derechos de las personas con discapacidad y de su inclusión social. Recuperado de https://bit.ly/1I72YFv.

Borland, J. y James, S. (1999) The Learning Experience of Students with Disabilities in Higher Education. A case study of a UK university. Disability \& Society, 14, 1, 85-101.

Castellana, M. y Sala, I. (2005). La Universidad ante la diversidad en el aula. Aula Abierta, 85, 57-84.

Castellana, M. y Sala, I. (2006). La inclusión de los estudiantes con discapacidad en la universidad: un reto para la universidad española en el nuevo espacio europeo de la educación superior. Aloma, 18. Consultado el 26-10-11, disponible en https://bit.ly/2JcUcQh.

Cotán, A. (2015). Enseñanza Superior y Educación Inclusiva: múltiples miradas desde las historias de vida de estudiantes con discapacidad. Tesis Doctoral: Universidad de Sevilla. Sevilla.

Cotán, A. (2016). ¿Un entorno que discapacita? Principales barreras de transporte e infraestructura identificadas en la historia de vida de Rafa. Kultur, 3(6), 195-214. doi: 10.6035/Kult-ur.2016.3.6.8

Declaración de Salamanca (2004). Conferencia Mundial sobre Necesidades Educativas Especiales: Acceso y Calidad. Disponible en: https://bit.ly/2XG2Hwz.

Dell, C.A., Dell, T.F., y Blackwell, T.L. (2015). Applying universal design for learning in online courses: pedagogical and practical considerations. The Journal of Educators Online-JEO, 13, 2, 166192.

European Commission (2010). Estrategia Europa 2020 para un crecimiento inteligente, sostenible e integrador (2014). Bruselas: Unión Europea.

Fernández, Mª.D., Álvarez, Q., y Malvar, Ma.L. (2012) Accesibilidad e inclusión en el Espacio Europeo de Educación Superior: el caso de la Universidad de Santiago de Compostela. Aula Abierta, 40(3), 71-82.

Frank, A. W. (2011). Practicing Dialogical Narrative Analysis. En J.A. Holstein y J. F. Gubrium (Eds.). Varieties of narrative analysis (33-52). Los Ángeles: Sage Publications.

Fuller, M., Bradley, A., y Healey, M. (2004). Incorporating disabled students within an inclusive higher education environment, Disability \& Society, 19, 5, 455-468

Fundación ONCE (2010). Estudio sectorial por comunidades autónomas de la accesibilidad del entorno universitario y su percepción. Observatorio Universidad y Discapacidad. Barcelona: Observatorio Universidad y Discapacidad

Fundación Universia (2018). Guía de atención a la discapacidad en la Universidad. Online: Universia. Recuperado de https://bit.ly/2GrEiSs (26 de febrero de 2019)

Goetz J.P. y LeCompte, M.D. (1988). Etnografia y diseño cualitativo en investigación cualitativa. Madrid: Morata.

Goodley, D., Lawthom, R., Clough, P., y Moore, M. (2004). Researching life stories. London: Routledge.

Hadjikakou, K., Polycarpou, V., y Hadjilia, A. (2010) The Experiences of Students with Mobility Disabilities in Cypriot Higher Education Institutions: Listening to their voices. International Journal of Disability, Development and Education, 57(4), 403-426. doi: 10.1080/1034912X.2010.524445.

Hanafin, J., Shevlin, M., Kenny, M., y McNeela, E. (2007). Including young people with disabilities: Assessment challenges in higher education. Higher Education, 54, 435-448. doi: 10.1007/s10734006-9005-9. 
Hopkins, L. (2011). The path of least resistance: a voice-relational analysis of disabled students experiences of discrimination in English universities, International Journal of Inclusive Education, 15, 7, 711-727. doi: 10.1080/13603110903317684.

Juncà, J. A. 2003. Buenas prácticas en accesibilidad universal. Ingeniería y Territorio, 63, 16-23.

Leíte, A.E. (2011). Preocupaciones epistemológicas y metodológicas en torno a la construcción de historias de vida. En F. Hernández, J.M Sancho, y J.I. Rivas (Coord). Historias de vida en educación. Biografias en contexto (pp.42-46). Barcelona: Esbrina.

López-Gavira, R. y Moriña, A. (2015). Hidden voices in higher education: inclusive policies and practices in social science and law classrooms. International Journal of Inclusive Education, 19(4), 365378. doi: $10.1080 / 13603116.2014 .935812$.

Luque, D.J., Rodríguez, G., y Romero, J.F. (2005). Accesibilidad y Universidad. Un estudio descriptivo. Intervención Psicosocial, 14, 2, 209-222.

Mace, R. (1997). About Universal Design. Center on Universal Design, North Carolina State University.

Mallimaci, F., y Giménez, V. (2006). Historias de vida y Método Biográfico. En Vasilachis de Gialdino, I. (Coordinadora) (2006). Estrategias de Investigación Cualitativa (175-212). Barcelona: Gedisa.

Meyen, E. (2015). Significant Advancements in Technology to Improve Instruction for all Students. Including those with Disabilities. Remedial and Special Education 36(2), 67-71. doi: 10.1177/0741932514554103.

Miles, M. B. y A. M. Huberman (1994). Qualitative Data Analysis. CA: Sage Publications.Moriña y Morgado (2018),

Moriña, A. (2017). Inclusive education in higher education: challenges and opportunities. European Journal of Special Needs Education, 32(1), 3-17. doi: 10.1080/08856257.2016.1254964.

Moriña, A. y Cotán, A. (2017). Educación Inclusiva y Enseñanza Superior desde la Mirada de Estudiantes con Diversidad Funcional. Revista Digital de Investigación en Docencia Universitaria, 11(1), 20-37. doi: http:10.19083/ridu.11.528

Moriña, A., Cortés, Mª D., y Melero, N. (2014). Inclusive curricula in Spanish higher education? Students with disabilities speak out. Disability \& Society. 29, 1, 44-57. doi: 10.1080/09687599.2013.769862.

Moswela, E. y Mukhopadhyay, S. (2011) Asking for too much? The voices of students with disabilities in Botswana. Disability \& and Society, 26, 3, 307-319. doi: 10.1080/09687599.2011.560414.

Naciones Unidas (1948. Declaración Universal de Derechos Humanos. Recuperado de https://bit.ly/23c5los (26 de febrero de 2019).

Naciones Unidas (2006). Convención sobre los Derechos de las Personas con Discapacidad y Protocolo Facultativo. Extraído de https://bit.ly/1pX3OTp (26 de febrero de 2019)

Oliver, M. y Barnes, C. (1998) Social Policy and Disabled People: From Exclusion to Inclusion. London: Longman.

Patton, M.Q. (1987). How to use qualitative methods in evaluation. London: Sage Publications.

Peralta, A. (2007). Libro Blanco sobre universidad y discapacidad. Madrid: Real Patronato sobre Discapacidad.

Pliner, S. y Johnson, J. (2004). Historical, theoretical, and foundational principles of universal instructional design in higher education. Equity \& Excellence in Education, 3, 105-113. doi: $10.1080 / 10665680490453913$.

Polo, $\mathrm{M}^{\mathrm{a}}$ T. y López, $\mathrm{M}^{\mathrm{a}}$ D. (2005). Barreras de acceso al medio físico de los estudiantes con discapacidad motora en la Universidad de Granada. Revista Electrónica de Investigación Psicoeducativa, 7, 3, 121-132. doi: 10.25115/ejrep.v3i7.1175.

Powell, J. J. (2013). From Ableism to Accessibility in the Universal Design University. The Review of Disability Studies: An International Journal, 8, 33-45. Recuperado de: https://bit.ly/2XdiChI.

Preiser, W. y Smith, K. (Eds.) (2011). Universal design handbook (2 nd Ed.). New York: McGrawHill.

Pujadas, J.J. (2002). El método biográfico: El uso de las Historias de Vida en Ciencias Sociales. Madrid: Centro de Investigaciones Sociológicas. 
Riddell, S., Tinklin, T., y Wilson, A. (2005). Disabled students in Higher Education. London: Routledge. Riessman, C. J. (2008). Narrative methods for the human sciences. Thousand Oaks, CA: Sage Publications. Rodríguez, A. y Álvarez, E., (2014). Estudiantes con discapacidad en la Universidad. Un estudio sobre su inclusión. Revista Complutense de Educación, 25, 2, 457-479. doi: 10.5209/rev RCED.2014.v25.n2.41683.

Rodríguez, P., Suso, A., Vázquez, D., y Velasco, Ma . L. (2009). Discapacidad, estudios superiores y mercado de trabajo. Barreras de acceso y repercusión en la inserción social. Madrid: Fundación Once.

Sandín, $\mathrm{M}^{a}$ P. (2003). Investigación Cualitativa en Educación. Fundamentos y Tradiciones. Madrid: McGraw Hill.

Sandoval, M., Simón, C., y Márquez, C. (2019): ¿Aulas inclusivas o exclutyentes?: barreras para el aprendizaje y la participación en contextos universitarios. Revista Complutense de Educación, 30(1), 261-276. doi: $10.5209 /$ RCED. 57266

Seale, J., Georgeson, J., Mamas, C., y Swain, J. (2015). Not the right kind of "digital capital"? Am examination of the complez relationship between disabled student, their technologies and higuer education institucions. Computers and Education, 82, 118-128. doi: 10.1016/i.compedu.2014.11.007.

Shevlin, M., Kenny, M., y Mcneela, E. (2004) Participation in higher education for students with disabilities: an Irish perspective, Disability \& Society, 19, 1, 15-30. doi: 10.1080/0968759032000155604.

Silver, P., Bourke, A., y Strehorn, K. C. (1998). Universal Instructional Design in higher education: An approach for inclusion. Equity \& Excellence, 31(2), 47-51. doi: 10.1080/1066568980310206.

Vander, M. (2015). Accessibility in Teaching Assistant Training: A Critical Review of Programming from Ontario's Teaching and Learning Centres. The Canadian Journal for the Scholarship of Teaching and Learning, 6, 2, Article 9. doi: 10.5206/cjsotl-rcacea.2015.2.9.

Watchorn, V., Larkin, H., Ang, S., y Hitch, D. (2013). Strategies and effectiveness of teaching universal design in a cross-faculty setting. Teaching in Higher Education, 18, 477-490. doi: $\underline{10.1080 / 13562517.2012 .752730 .}$.

\section{Para citar este artículo}

Cotán Fernández, A. (2019). Hábito lector en estudiantes de Primaria: influencia familiar y del Plan Lector del centro escolar. Revista Fuentes, 21(1), 85-101. [Fecha de consulta: dd/mm/aa]. doi: 10.12795/revistafuentes.2019.v21.11.06 